\title{
HIGH POWER DISK LOADED GUIDE LOAD *
}

\author{
Z. D. Farkas, SLAC, Stanford, CA 94025, USA.
}

\begin{abstract}
A method to design a matching section from a smooth guide to a disk-loaded guide, using a variation of broadband matching, $[1,2]$ is described. Using this method, we show how to design high power loads. The load consists of a disk-loaded coaxial guide operating in the $\mathrm{TE}_{01}$-mode. We use this mode because it has no electric field terminating on a conductor, has no axial currents, and has no current at the cylinder-disk interface. A high power load design that has $-35 \mathrm{~dB}$ reflection and a $200 \mathrm{MHz},-20 \mathrm{~dB}$ bandwidth, is presented. It is expected that it will carry the $600 \mathrm{MW}$ output peak power of the pulse compression network. We use coaxial geometry and stainless steel material to increase the attenuation per cell.
\end{abstract}

\section{INTRODUCTION}

Although the main focus of this note is on a high power load, this method of broadband matching will also be applied to filters and attenuators [3]. A magnetic stainless steel load using a $\mathrm{TE}_{11}$-mode in a round guide has been developed and implemented [4]. Here we present a load that uses a $\mathrm{TE}_{01}$-mode travelling wave in a disk-loaded guide (DLG). This mode has

- No electric field terminating on a conductor, hence no field emission.

- No axial currents and no axial electric field.

- No currents at the waveguide-disk interface.

Thus, it is expected to have higher average and peak power carrying capacity. The peak magnetic fields, which limits peak power, will be lower. Also, this load, composed of $\mathrm{TE}_{01}$ mode cells, does not require brazing, which may cause a reduction of permeability, and hence reduce the attenuation. In order to maximize the bandwidth (BW), the phase advance per cell is $\pi / 2$.

\section{DISK LOADED MATCHING SECTION THEORY}

We match from a smooth guide to a disk-loaded guide. To obtain a wide bandwidth, the matching is based on a well known design [1,2], which stipulates $\pi / 2$ line lengths whose impedances vary so that the input reflection coefficient, $\Gamma_{i n}$, is a Butterworth or a Tchebychev polynomial. In our design, the matching $\pi / 2$ transmission-line lengths are replaced by $\pi / 2$ phase advance disk-loaded cells and

\footnotetext{
* Work supported by the Department of Energy, contract DE-AC02-
} $76 \mathrm{SF} 00515$ their characteristic impedance is replaced by the travelling wave impedance, defined as

$$
Z_{t}=\frac{E^{2}}{P_{t}}=\left(1 / v_{g}\right) E^{2} / w .
$$

$E$ is the maximum electric field $P_{t}$ is the transmitted power, and $w$ is the energy stored per unit length. $Z_{t}$ has units of ohms per square meter. Figure 1 shows impedance matching with $90^{\circ}$ transmission line sections, (similar to Figure 4.1.4 in reference [2]), and the equivalent matching with $90^{\circ}$ phase advance disk-loaded cells. In both cases their impedances are such that the input reflection coefficient to the matching network, $\Gamma_{i n}$, is a Butterworth or a Tchebychev polynomial.
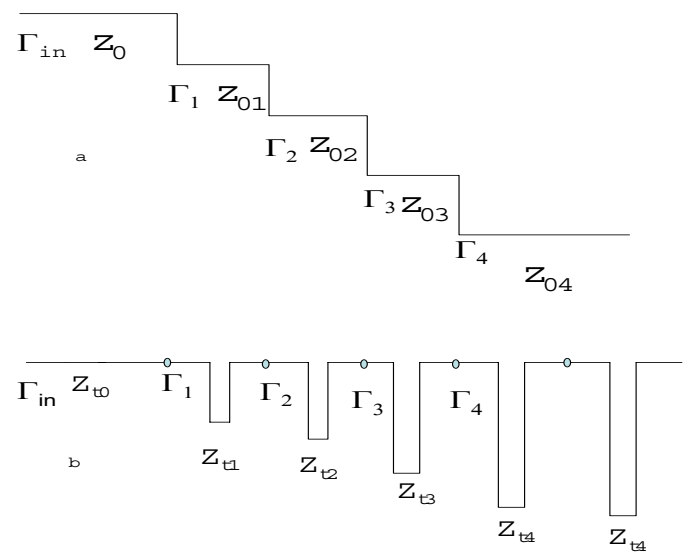

Figure 1: a) Impedance matching with $90^{\circ}$ transmission line sections and $\mathrm{b}$ ) the equivalent matching with $90^{\circ}$ phase advance disk-loaded cells.

Choose the number of matching cells, $N_{m}$. The number of reflections (discontinuities) is $N_{r}=N_{m}+1$. Choose the reflection coefficient of the first cell, $\Gamma(1)$. Its value is a trade-off between bandwidth and length of the load. The remaining reflection coefficients are given by

$$
i=2, \ldots N_{r}, \quad \Gamma(i)=r_{\gamma}(i) \times \Gamma(1),
$$

where $r_{\gamma}(i)$, are the reflection coefficient ratios, which are binomially distributed so that $\Gamma_{i n}$ is a Butterworth function [2]. The higher the value of $\Gamma(1)$ and the subsequent $\Gamma$ 's, the higher the impedances and the lower the group velocities of the cells. Consequently the attenuation is higher and the load is shorter. But the bandwidth is narrower and the energy densities and fields are higher. The ratio of the impedances, which are also the standing wave ratios (SWR), 
are:

$$
\rho(i)=\frac{1+\Gamma(i)}{1-\Gamma(i)} \quad i=1,2, \ldots N_{r}
$$

As we are interested in ratios only, we normalize to the travelling wave impedance of the input guide, $Z_{t 0}$. $Z_{t n} \equiv$ $Z_{t} / Z_{t 0}$. The normalized impedances required for matching are:

$Z_{t n}(1)=\rho(1), Z_{t n}(i)=Z_{t n}(i-1) \rho(i-1), i=2, \ldots N_{r}$.

To increase losses we use coaxial geometry. We choose the outer conductor radius $r_{\text {out }}=2.851 \mathrm{~cm}$, inner conductor radius $r_{i n}=0.953 \mathrm{~cm}$ and disk thickness $t_{d}=0.5 \mathrm{~cm}$. At $11,424 \mathrm{MHZ}$, the TW impedance, and the group velocity are, respectively,

$$
Z_{t 0}=35.14 \mathrm{Mohm} / \mathrm{m}^{2}, v_{g 0} / c=69.6 \%,
$$

We choose $\Gamma(1)=0.16$. We choose $N_{m}=3$, hence the impedance ratios, $r_{\gamma}=\left[\begin{array}{llll}1 & 3 & 3 & 1\end{array}\right]$. Using equations 2 through 4 , we obtain the normalized impedances, $Z_{t n}$, of the four cells ( 3 cells required for matching plus the first attenuation cell). A typical coaxial cell and a waveguide cell are shown in Figure $2 \mathrm{a}$ and b, respectively. Frequency vs. phase advance ( $\omega-\beta$ diagram) and vs. group velocity for several cells is also shown in Figure 2.
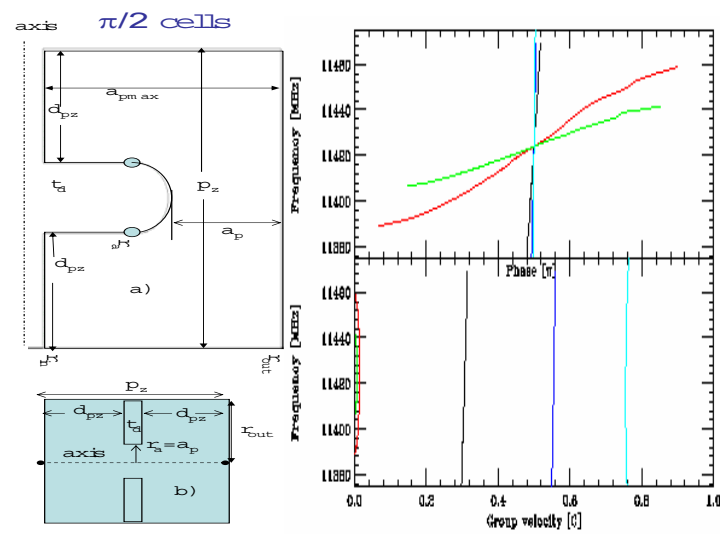

Figure 2: Cell cross sections and frequency vs. phase advance and vs. group velocity.

Color plots of the electric and magnetic fields, in a 45 degree wedge of a typical cell, are shown in Figure 3. A method of obtaining the required values of $Z_{t n}$, using HFSS and SMART2D [5] are described in reference [3]. The length of a cell (spacial period) is $p_{z}=2 d_{p z}+t_{d}$ where $t_{d}$ is the disk thickness. The aperture and the maximum aperture are:

$$
a_{p}=r_{\text {out }}-r_{a}-t_{d} / 2, \quad a_{\text {pmax }}=r_{\text {out }}-r_{\text {in }} .
$$

At the maximum aperture (no disk):

$$
\lambda_{g}=\lambda_{g 0}, \quad p_{z}=p_{z 0}=\lambda_{g 0} / 4, \quad v_{g}=v_{g 0} .
$$

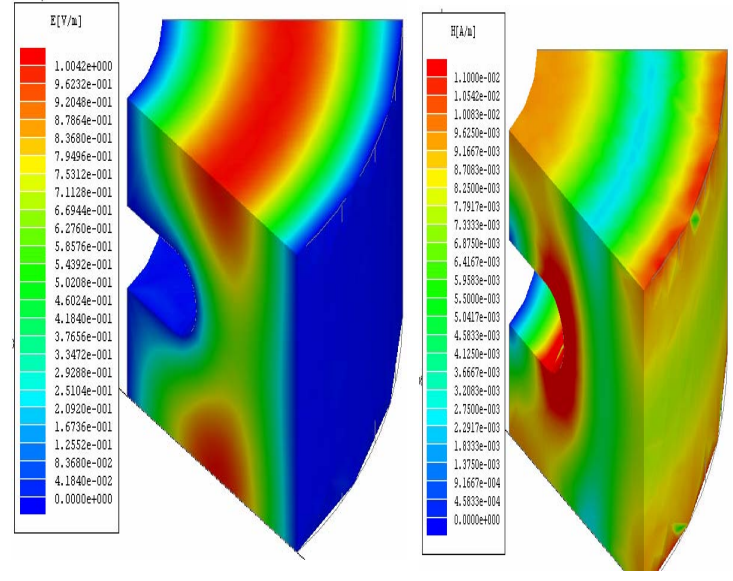

Figure 3: A 45 degree wedge of a typical cell showing electric and magnetic fields. $\mathrm{P}=36$ watts.

Define the normalized apertures and cell lengths as:

$$
a_{p n}=a_{p} / a_{p \max }, \quad p_{z n}=p_{z} /\left(\lambda_{g 0} / 4\right) .
$$

The distances between the disks are

$$
d_{c}(i)=d_{p z}(i)+d_{p z}(i+1), \quad i=1 \ldots N_{t}-1 .
$$

$N_{t}$ is the total number of disks.

\section{High Power Load}

To make a load, we add to the matching cells a sufficient number of attenuation cells to decrease the input reflection (return-loss) to an acceptable level and terminate the last attenuation cell with a short circuit. The distance between the last disk and the end short is not crucial. The length of the load depends on the attenuation per cell which is given by

$$
\alpha_{c}=\frac{p_{z} \pi f}{Q v_{g}} \text { nepers . }
$$

To increase the attenuation per cell (to make the load shorter) and to distribute the power dissipation more evenly, the group velocities, hence the apertures, are monotonically decreased by small amounts. The parameters of the attenuation cells can vary by small amounts. Here we linearly increased $r_{a}$ by $\Delta r_{a}=0.15 \mathrm{~mm}$. To connect the coax to a $\mathrm{TE}_{10}$ rectangular guide we can use a Wraparound Mode Converter(WMC) [6], which converts $\mathrm{RF}$ signals from the $\mathrm{TE}_{10}$ mode in rectangular guide to the $\mathrm{TE}_{01}$ mode in round guide. This has the advantage that the inner conductor can be secured at both ends. But a WMC limits input power and bandwidth, therefore the load has to have a broadband waveguide input. For testing the load at low power, a WMC for 1.5 inch diameter round guide is available. Therefore we design a matching section from 1.5 inch diameter round guide to the coax. We connect the waveguide to coax matching section to the coax input load to form a load with 1.5 inch diameter waveguide input. 


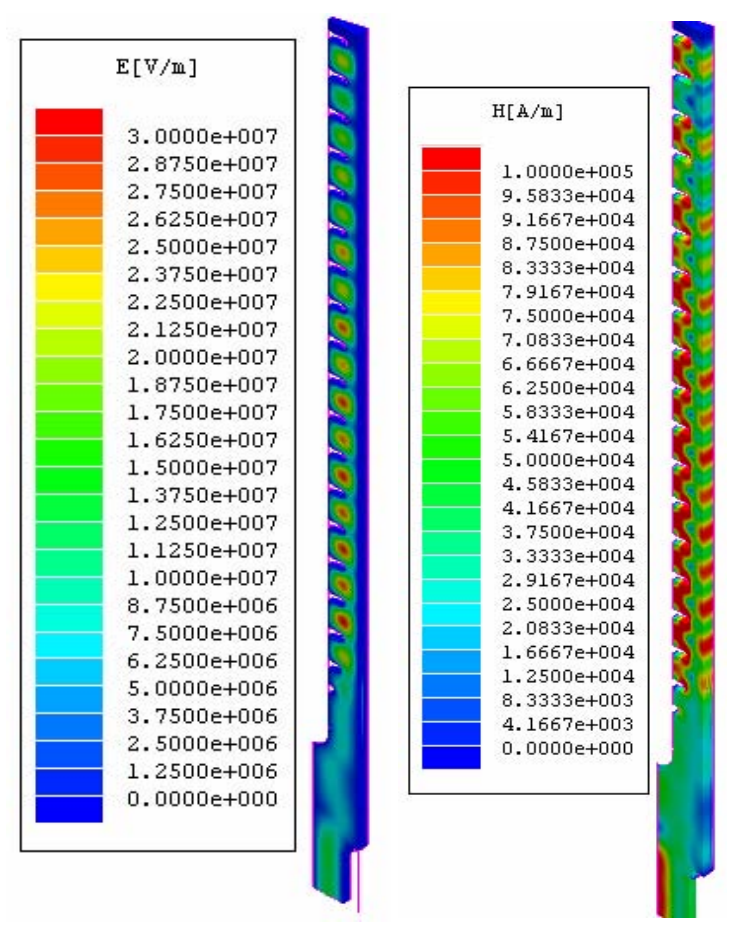

Figure 4: Color plots of electric and magnetic fields.

Color plots of the electric field and the magnetic field are shown in Figure 4. The input power is $100 \mathrm{MW}$. The resistivity and permeability relative to copper are 5.17 and 11 , respectively. Line plots of the electric field at $r=1.8 \mathrm{~cm}$, and

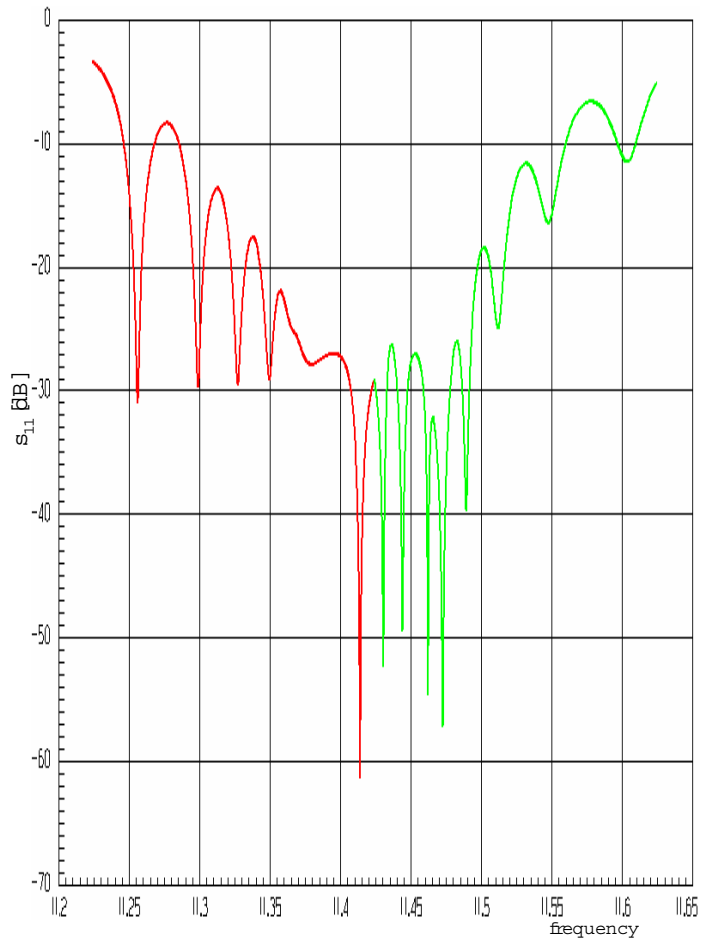

Figure 6: Reflection as a function of frequency.

the magnetic field at $r=r_{i n}$ are shown in Figure 5. The reflection, $s_{11}$ as a function of frequency shown in Figure 6.

\section{REFERENCES}

[1] S.B. Cohn, "Optimum Design of Stepped Transmission Line Transformers”, IRE Trans, Vol. MTT-3, pp 16-21, April, 1995.

[2] J. L. Altman, "Microwave Circuits", Ch. 4, Van Nostrand, 1964.

[3] Z. D. Farkas, “ $\mathrm{TE}_{01}$ High Power Disk Loaded Guide Load”, SLAC-TN-04-075.

[4] S. G. Tantavi, R . Vlieks "Compact X-band High Power Load Using Magnetic Stainless Steel”, PAC 1995.

[5] V.A. Dolgashev, "Calculation of Impedance for Multiple Waveguide Junction Using Scattering Matrix Formulation”, icap98

[6] S. G. Tantavi et. al. "The generation of $400 \mathrm{MW}$ RF pulses at X-band etc", IEEE Trans, Vol. MTT-47, pp 2539-46, Dec. 1999.

\section{ACKNOWLEDGEMENT}

I am grateful to V. Dolgashev and C. Nantista for their help.

Figure 5: Line plots of electric and magnetic fields. 